Relations industrielles

Industrial Relations

\title{
Décisions rendues par le Conseil canadien des relations du travail
}

\section{Catherine Saint-Germain}

Volume 41, numéro 2, 1986

URI : https://id.erudit.org/iderudit/050212ar

DOI : https://doi.org/10.7202/050212ar

Aller au sommaire du numéro

Éditeur(s)

Département des relations industrielles de l'Université Laval

ISSN

0034-379X (imprimé)

1703-8138 (numérique)

Découvrir la revue

Citer cet article

Saint-Germain, C. (1986). Décisions rendues par le Conseil canadien des relations du travail. Relations industrielles / Industrial Relations, 41(2), 397-411. https://doi.org/10.7202/050212ar
Résumé de l'article

Decisions rendues par le conseil canadien des relations du travail
Tous droits réservés (C) Département des relations industrielles de l'Universite Laval, 1986
Ce document est protégé par la loi sur le droit d'auteur. L'utilisation des services d'Érudit (y compris la reproduction) est assujettie à sa politique d'utilisation que vous pouvez consulter en ligne.

https://apropos.erudit.org/fr/usagers/politique-dutilisation/ 


\section{Décisions rendues par le Conseil canadien des relations du travail}

\section{Accréditation dans le secteur bancaire}

Le Conseil a rendu une décision sans précédent en accordant une accréditation visant tous les employés de toutes les succursales de la Banque Nationale à Rimouski. Dans ses motifs, le Conseil trace une gigantesque fresque de l'histoire et des aléas du mouvement syndical dans ce secteur.

Syndicat des employés des Banques Nationales de Rimouski (CSN), requérant, et Banque Nationale du Canada, employeur.

Dossier du Conseil 555-1685, décision du 18 décembre 1985, (No 542); Panel du Conseil: Me Marc Lapointe, c.r., Président, MM. Jacques Archambault et Victor E. Gannon, Membres. Motifs rédigés par M. Marc Lapointe.

\section{FAITS SAILLANTS}

Le Syndicat des employés des Banques Nationales de Rimouski (CSN) demandait que lui soit accordée l'accréditation d'une unité de négociation comprenant les employés des cinq succursales de la Banque Nationale situées à Rimouski.

La Banque Nationale, issue de la fusion récente de la Banque Canadienne Nationale et de la Banque Provinciale, exerçait ses activités à travers l'ensemble du Canada, quoique de façon plus concentrée au Québec. Les structures opérationnelles de la Banque se composaient de huit divisions administratives, dont six situées au Québec. Dans la vaste Division VI, couvrant le Bas St-Laurent, la Banque administrait environ cent succursales qu'elle avait rassemblées en cinq groupes; dans le groupe 64, formé de vingt établissements se dispersant de la rive sud du St-Laurent jusqu'à la Baie des Chaleurs en passant par la Gaspésie, on retrouvait les fameuses cinq succursales de Rimouski que le syndicat voulait englober dans une même unité.

Également, la Banque avait établi sept zones salariales qui ne coincidaient pas avec les Divisions. La zone I, en sus de tout le Québec, comprenait les quatre provinces de l'Atlantique, une partie du Manitoba et une partie de l'Ontario.

La preuve a aussi révélé que Rimouski était un petit centre urbain, caractérisé par un isolement relatif, et qu'avec sa région immédiate, il était beaucoup plus dynamique que le reste du territoire l'entourant.

- Cette chronique a été rédigée par Catherine SAINT-GERMAIN, avocate, conseiller juridique auprès du Président du Conseil canadien des relations du travail.

Toute opinion pouvant découler de la présente chronique et exprimée par l'auteur en sus du texte officiel des décisions du C.C.R.T., ne lie pas ce dernier. 
Quant aux employés des cinq succursales, ils n'avaient pour la plupart jamais travaillé ailleurs que dans l'un de ces établissements. Cependant, certaines activités de la Banque entraînaient des échanges d'employés d'une succursale à l'autre, à Rimouski.

\section{QUESTIONS SOULEVÉES}

Une grappe de succursales situées dans une même ville, en l'occurrence Rimouski, constitue-t-elle une unité de négociation appropriée lorsque l'employeur a des activités pan-canadiennes?

\section{PRINCIPAUX MOTIFS DE DÉCISION}

Avant d'examiner le caractère approprié ou non de l'unité proposée, le Conseil s'est attaché à passer en revue sa jurisprudence, ainsi que celle d'autres juridictions, et à faire ressortir les faits qui sont à l'origine du si faible taux de syndicalisation $(1.4 \%$ au Canada) des employés des institutions financières. À l'appui de sa narration, il a annexé à sa décision une volumineuse documentation qui fait partie intégrante des motifs.

\section{La jurisprudence du Conseil}

En 1959, dans l'affaire Bank of Nova Scotia, Kitimat, ${ }^{1}$, le Conseil d'alors avait rejeté la demande d'accréditation visant une succursale distincte en raison de l'apparence non-viabilité de l'unité proposée et de son inhabilité à négocier. Mais il prenait pourtant soin de ne pas fermer la porte à ce type d'accréditation.

Même si cette requête est rejetée, le Conseil estime qu'il est opportun d'affirmer que la présente décision ne doit pas être considérée comme une indication qu'il est d'accord avec l'affirmation de l'intimée selon laquelle l'unité de négociation appropriée est celle qui regrouperait tous les employés de la banque répartis dans tout le pays. La présente décision est fondée sur des circonstances particulières... ${ }^{2}$

Le Conseil fait ensuite état de la cause Banque d'Épargne de la Cité et du district de Montréal, ${ }^{3}$ où son prédécesseur décerna un certificat d'accréditation visant tous les employés des succursales et du bureau chef de cette banque. La décision, cependant, demeure aussi particulière.

Cependant, l'examen des circonstances entourant ce dossier permet de conclure qu'il s'agissait d'un accident de parcours, si on peut nous permettre cette

1 (1959), 59 CLLC 18,152.

2 Idem; (traduction) p. 1799.

3 Décision non publiée du 6 octobre 1967, dossier 7-66-1936. 
expression. En effet, il ne s'agissait pas d'une banque majeure, pas même d'une banque d charte. Non seulement minuscule en importance et établie en la seule province de Québec, elle n'opérait que dans la région de la Cité de Montréal et comptait seulement 75 succursales. Et, comme son nom l'indique, l'aire de ses opérations financières était réduite. ${ }^{4}$

Une décennie plus tard, la décision de ce Conseil dans Banque canadienne impériale de commerce ${ }^{5}$ eut l'effet d'une bombe en déclarant appropriée une unité ne comprenant qu'une succursale. Deux syndicats non affiliés aux grandes centrales syndicales avaient déposé plusieurs requêtes visant des succursales individuelles, l'un en Ontario et l'autre en Colombie-Britannique. Après une étude extensive de l'industrie bancaire ayant duré 7 mois, le Conseil décernait les certificats demandés. Ces décisions déclenchèrent une flambée d'organisation syndicale dans les banques au Canada et firent couler beaucoup d'encre. Mais ce fut là bien du branle-bas pour peu de conquêtes. Un des syndicats ainsi accrédité, abandonnait ses certificats un an plus tard, impuissant à pénétrer davantage la forteresse qu'était la Banque canadienne impériale de commerce et ceux qui obtinrent par la suite de telles accréditations ne purent obtenir des employeurs que des concessions mineures, alors que les banques procédaient à une flopée d'améliorations aux conditions de travail dans leurs succursales non syndiquées. Une véritable guérilla juridique s'établissait et le Conseil passe en revue sa jurisprudence relative aux plaintes de pratiques déloyales qui engorgèrent ses rôles d'auditions ${ }^{6}$.

Quoiqu'il en soit, le mouvement syndical crut, à tort, comme en témoigne la présente décision, que le Conseil avait cristallisé l'unité appropriée sous la forme d'une succursale distincte.

De 1977 à 1982 (date de la requête qui fit l'objet de la présente décision), il n'y eut qu'une demande syndicale visant un autre type d'unité. Banque de Montréal (Windsor) ${ }^{7}$. Le syndicat demandait. alors au Conseil de grouper plusieurs accréditations individuelles en les consolidant en une seule et d'y inclure au surplus les

4 Décision originale, page 7.

5 (1977), 20 di 319; [1977] 2 Can LRBR 99; 77 CLLC 16,089.

6 Banque de Nouvelle-Écosse (Selkirk Man.) (1978), 27 di 696; [1978] 1 Can LRBR 544; Banque Royale du Canada (Kamloops et Gibsons) (1978), 27 di 701; [1978] 2 Can LRBR 159; 78 CLLC 16,132; Banque de Nouvelle-Ecosse (Vancouver Heights) (1978), 28 di 901; [1978] 2 Can LRBR 181; Banque canadienne impériale de commerce (Gibsons, C.-B.) (1978), 27 di 748; Banque canadienne impériale de commerce (Sioux Lookout), (1978), 33 di 432; [1979] 1 Can LRBR 18; Banque canadienne impériale de commerce (succursales North Hills et Victoria Hills (1979), 34 di 651; [1979] 1 Can LRBR 266; 80 CLLC 16,001; Banque canadienne impériale de commerce, (1979) 134 di 677; [1979] 1 Can LRBR 391; Banque canadienne nationale (1979), 35 di 39; Banque canadienne impériale de commerce (Creston et St. Catherines) (1979), 35 di 105; [1980] 1 Can LRBR 307; 80 CLLC 16,002; Bank of British Columbia (1980), 41 di 188; [1980] 3 Can LRBR 576; 81 CLLC 16,068; Banque Royale du Canada (1980), 42 di 125; Banque de Nouvelle-Écosse (Sherbrooke et Rock Forest) (1980), 42 di 216; [1981] 2 Can LRBR 365; 81 CLLC 16,110; Banque Nationale du Canada (1981), 42 di 352; [1982] 3 Can LRBR 1; Banque de Nouvelle-Écosse (Sherbrooke et Rock Forest) (1982), 42 di 398; [1982] 2 Can LRBR 21; 82 CLLC 16,158; Banque de Montréal (succursale Devonshire Mall) (1982), 51 di 160; 83 CLLC 16,015; Canadian Imperial Bank of Commerce (1985), 85 CLLC 16,021.

7 (1981), 45 di 266; [1982] 2 Can LRBR 380. 
employés d'autres succursales syndiquées de la même banque. Mais à l'audition, il retira cette dernière partie de sa demande. La requête fut rejetée, soupçonnée d'être plus une manoeuvre de négociation collective que d'organisation syndicale. Toutefois, le Conseil n'avait pas exclu qu'une autre unité que celle d'une seule succursale puisse être une unité appropriée.

Mais il (le Conseil) tient à rectifier les conclusions de certains commentateurs sur un point et à souligner un aspect bien spécifique de la preuve dans l'une de ces deux décisions initiales.

$\grave{A}$ aucun moment les procureurs des banques n'adoptèrent-ils une position différente en 1976 de celle qu'ils avaient plaidée en 1959 dans la cause Bank of Nova Scotia, Kitimat, supra.

... Il ne pouvait y avoir qu'une seule unité appropriée dans les banques canadiennes: tous les employés de toutes les succursales d'une banque, ou rien.

Et ce, après avoir longtemps argumenté que les banques étant d'une telle importance vitale dans l'ossature de la nation qu'il était presque inconcevable que leurs employés puissent accéder à la négociation collective avec ses possibilités de chaos à l'occasion d'arrêts de travail.

Et du côté syndical, à aucun moment leurs procureurs n'adoptèrent-ils sérieusement une position différente en 1976 de celle qui avait donné lieu à la requête dans la cause Bank of Nova Scotia, Kitimat, supra. Une succursale constituait une unite appropriée. $)^{8}$

«Le Conseil avait maintes fois expliqué son rôle, tel qu'il le percoit da la lumière du statut qu'il administre et interprète et des pouvoirs qui lui sont dévolus. Il réagit à des requêtes qui doivent originer en général des parties.

On lui propose une unité donnée comme habile à négocier dans des circonstances bien précises. Il la sanctionne, ou non, modifiée ou non. Lui en demander plus suffirait a le faire taxer d'interventionniste. ${ }^{9}$

\section{La jurisprudence américaine}

Le Conseil passe également en revue la jurisprudence américaine pour constater que celle-ci a progressivement fait naître la présomption que l'unité de succursale individuelle serait appropriée (splinter approach) ${ }^{10}$. Cependant, il conclut aussi que le concept de «grappe» (cluster), a fait son apparition aux Etats-Unis dans un secteur aux structures administratives similaires à celle de l'industrie bancaire, soit les chaînes de pharmacies ${ }^{11}$.

8 Supra, note 4, pp. 20, 21.

9 Idem, p. 24.

10 Hawaii National Bank, Honolulu, 212 NRLB 576 (1974); State Bank of India, 95 LRRM 1141 (1977); Rocky Mountain Bank Note Company, 230 NLRB 922 (1977); Wyandotte Savings Bank, 102 LRRM 1349 (1979); Alaska State Bank, 105 LRRM 1055 (1980); The Exchange Bank, 111 LRRM 1602 (1982).

11 Gray Drug Stores Inc., 1972 CCH NLRB 24,345; Hagg Drug Company, 169 NLRB 877 (1968; Mott's Shop Rite of Springfield Inc. and Mott's Shop Rite of Chicopee Inc., 182 NLRB 172 (1970). 
Les circonstances particulières au secteur bancaire

Le Conseil s'est attentivement penché sur des études sociologiques (dont les extraits annexés à la décision font partie intégrante des motifs) identifiant les raisons qui sont à l'origine du si faible taux de syndicalisation (1.4\%) des employés du secteur des institutions financières au Canada. Il a ainsi retracé quatre principales causes de cet état de fait.

Tout d'abord, les employés de banque font partie des "cols blancs», soit la catégorie qui constitue traditionnellement un faible pourcentage de la clientele syndicale. Ceci s'explique par le fait que leurs tâches comportent des conditions physiques souvent plus agréables que chez les cols bleus et qu'ils partagent une certaine communauté d'intérêts avec la direction en raison de leur proximité physique avec celle-ci et de leur statut social. Ils bénéficient également d'une plus grande sécurité d'emploi que les cois bleus (quoiqu'au niveau des banques, la révolution technologique engendrée par l'informatique rend aléatoire ce dernier point).

Le personnel des banques est majoritairement féminin. Les femmes seraient moins militantes et leur condition sociale les rend plus enclines à travailler à temps partiel, ou occasionnellement, et ce personnel instable à haut taux de roulement est difficile à syndiquer.

Les structures administratives des banques, soit un réseau particulièrement complexe de bureaux-chef et surtout de nombreuses succursales, peuplées d'un nombre restreint d'employés et réparties à l'échelle du Canada, rendent ardue la définition d'une unité appropriée. En ce sens, le partage constitutionnel des compétences n'a pas rendu les choses plus faciles car les «presque-banques», soit les diverses institutions financières de juridiction provinciale, ont été plus aisément pénétrées par le mouvement syndical.

Enfin, l'attitude farouchement anti-syndicale des banques à charte, parées de puissantes ressources, a aussi contribué à ralentir le processus de syndicalisation.

Mais le Conseil a fait également remarquer que les récentes transformations technologiques, soit principalement l'informatique, tendent à catalyser une certaine activité syndicale:

... un facteur commun à toutes les catégories d'employés... fut de rendre une grande partie du travail, quoique beaucoup plus efficace, par ailleurs plus répétitif et plus monotone... l'informatisation d'une foule de catégories d'employés cléricaux ou cols blancs... pourrait se solder par des réductions de personnel parmi des employés de catégories bien établies et bien connues dans plusieurs secteurs des industries de services, y compris celles des institutions bancaires et financières et parmi certaines de ces classifications on pourrait retrouver celle des caissières. ${ }^{2}$ 
Dans sa détermination de l'unité appropriée, le Conseil a tenu compte de ces circonstances difficiles pour le mouvement syndical:

Somme toute, par cette décision le Conseil entend, encore une fois, poursuivre sa politique d'exercer le pouvoir qui lui a été conféré par le paragraphe 125(2) du Code, de manière à permettre aux employés de banque, dans la mesure du possible, «d'avoir une possibilité réelle de faire valoir leurs droits en vertu du Code." (Voir La Banque Royale du Canada, supra, p. 2.) ${ }^{13}$

\section{Détermination de l'unité}

Après avoir étudié les structures administratives de la Banque Nationale (telles que décrites plus haut sous "faits saillants»), le Conseil établit d'abord que le concept d'un groupement de négociation n'est pas statique. De plus, il réitère les principes posés par sa jurisprudence antérieure:

Il (le Conseil) a établi d'une façon assez formelle les limites extrêmes de l'exercice de sa discrétion à cet égard, de la façon suivante:

1. Il n'est pas tenu de déterminer l'unité idéale

2. Il peut y avoir plus qu'une unité appropriée, successivement ou simultanément

3. Il n'est pas lié par une détermination antérieure et il peut en changer.

Il ne lui sera pas toujours possible de déterminer l'unité la plus appropriée et ce, pour une foule de raisons, dont la plus importante, sans doute, serait que cette détermination aurait pour effet de priver un groupe d'employés de leur liberté d'opter pour une association de leur choix (article $110 \mathrm{du}$ Code).

D’une façon générale, les unités idéales, pour des raisons déjà expliquées dans une longue jurisprudence, sont celles qui regroupent le plus d'employés possible. Mais cela n'est pas toujours possible et ne l'est certainement pas dans le secteur bancaire canadien avec ses structures bien caractéristiques, décrites, dans les présents motifs. ${ }^{14}$

Puis le Conseil statue que, si il est préférable d'épouser les structures fonctionnelles de l'employeur, cela n'est pas toujours possible:

Ce Conseil l'a déjà dit et croit encore qu'il faut tenir compte des structures administratives d'un employeur tant que faire se peut et en autant que ce critère ne rend pas impossible l'exercice du droit au choix libre d'une association par un groupe d'employés. Serait-ce possible chez la Banque Nationale du Canada pour le groupe d'employés qui ont opté pour fonder le syndicat requérant ?15 $^{15}$

\footnotetext{
13 Idem, p. 78.

14 Idem, p. 69.

15 Idem, pp. 70-71.
} 
Le Conseil répond par la négative. Calquer une unité sur la structure des Divisions ou des zones salariales est impensable. Ces territoires sont trop vastes. Même se baser sur la structure des groupes, qui est plus restreinte géographiquement, aboutirait également à une impasse:

La communauté d'intérêts entre les caissières de Rimouski et celles de Gaspé risque de ne pas survivre aux frais engagés par le petit syndicat de Rimouski obligé de défrayer des assemblées générales par la largeur de la Gaspésie. ${ }^{16}$

Finalement, le Conseil conclut que dans un pays aussi vaste et dépeuplé que le Canada, où l'on trouve des rassemblements de population isolés, le concept de la grappe (cluster) est un type d'unité appropriée. Il s'agit là d'une unité géographique, mais qui ne doit pas être entièrement assimilée à une unité de ville.

Que dire de cette unité nouvelle? On a vu dans la jurisprudence du NLRB aux États-Unis, que le «splinter» (succursale individuelle) est la règle. Mais dans une industrie où l'on retrouve un employeur à multiples succursales, l'industrie pharmaceutique, on a inventé le concept du «cluster» ou «grappes» d'établissement...

Nous préférons ce concept à celui d'unité appropriée de succursales de ville. Nous ne pouvons que croire qu'il existe plusieurs communautés au Canada, pays aussi vaste que dépeuplé, où l'on retrouve des rassemblements de population isolés mais réunissant les mêmes caractéristiques que la ville de Rimouski au Québec...17

Également, au vu de la prolifération actuelle des changements technologiques, le Conseil se prononce pour la création d'unités verticales qui seules, dans le secteur bancaire, pourront survivre.

L'argument de l'employeur à l'effet qu'une telle unité permettrait à une grosse succursale d'imposer sa volonté à une plus petite est rejeté:

Nous ne pouvons accepter cette dialectique qui tend à nier tout le système canadien et nord-américain de l'accréditation. ${ }^{18}$

Il ne s'agit pas là, selon le Conseil, d'un changement de cap. Au contraire, cette décision s'inscrit dans le cadre de sa politique bien définie:

... permettre aux employés de banque d'avoir une possibilité réelle de faire valoir leurs droits en vertu du Code. ${ }^{19}$

\section{DÉCISION}

Le Conseil accorde la requête du syndicat et accrédite ce dernier pour représenter tous les employés des cinq succursales de la Banque Nationale à Rimouski ${ }^{20}$.

16 Idem, p. 72.

17 Idem, p. 76.

18 Idem, p. 75.

19 Supra, note 13.

20 Une requête en révision judiciaire de cette décision est actuellement pendante devant la Cour fédérale du Canada, dossier de la Cour: A-1008-85. 


\section{Règlement des modalités d'une première convention collective dans le secteur bancaire}

Encore une fois, le Conseil a établi un précédent dans le secteur bancaire en réglant les modalités de la première convention collective entre la Banque de commerce canadienne impériale et le Syndicat des employés de banque visant deux unités de négociation distinctes, ceci dans la poursuite de l'objectif fondamental du Code canadien du travail: promouvoir et faciliter la négociation collective.

Le Syndicat des employés de banque (Ontario), section locale 2104, Congrès du travail du Canada, agent négociateur accrédité, et la Banque de commerce canadienne impériale, employeur.

Dossier du Conseil 675-18, décision du 27 janvier 1986, (N 564); Panel du Conseil: M. Hugh R. Jamieson, Vice-président, M. Victor E. Gannon et Mme Linda M. Parsons, Membres, motifs rédigés par M. Hugh R. Jamieson.

\section{FAITS SAILLANTS}

Le Syndicat avait été accrédité en septembre 1984 et en mars 1985 pour représenter respectivement les employés du Centre Chargex et ceux du Service central de courrier.

Les deux unités étaient en grève depuis plusieurs mois lorsque le Ministre renvoya l'affaire au Conseil pour faire une enquête sur le différend et, le cas échéant, régler les modalités d'une première convention entre les parties, en vertu de l'article 171.1 du Code canadien du travail. L'enquête du Conseil s'étendit du 30 décembre 1985 au 21 janvier 1986, incluant seize jours d'audition. Les témoignages, qui concernèrent surtout l'affaire du Centre Chargex, révélèrent que dès la première rencontre, les parties aux négociations se retrouvaient en polémique, la Banque contestant la qualité du comité de négociation syndical; il fallut onze rencontres pour que cette controverse soit réglée.

L'intervention d'un conciliateur conduit à une entente quant aux clauses touchant les tableaux d'affichage, la reconnaissance du Syndicat par la Banque, la clôture et les conditions relatives aux grèves et aux lock-out. C'est toutefois le Syndicat qui avait fait les concessions. En fait, les témoignages révélèrent que la stratégie de négociation de la Banque consistait à adopter une attitude évasive pour gagner du temps et étirer les négociations le plus longtemps possible. Les seules "concessions» touchaient des points déjà standardisés par la loi: avis minimum en cas de mises a pied, subordination des grèves et lock-out à certaines conditions, arbitrage des griefs, reconnaissance du Syndicat.

Mais la Banque se montrait intraitable lorsqu'il s'agissait de changer le statu quo quant aux questions touchant les avantages ou son droit de transiger directement avec les employés. Elle avait même déclaré publiquement qu'un changement à sa politique risquerait d'étendre d'avantage le mouvement de syndicalisation. En mai 1985, après 4 mois de négociations, alors qu'un consensus avait été atteint seulement 
quant à quelques questions accessoires, et ce, toujours suite à un compromis du Syndicat, la Banque déposait à la table une offre globale, qui s'avéra n'être rien de plus que le statu quo. De plus, la Banque tenta de négocier directement avec ses employés à plusieurs reprises, dans le but évident d'influencer le résultat d'un vote de grève imminent; en vain, car le Syndicat déclenchait une grève le 12 juin 1985. Ayant prévu que le conflit serait ardu, il annonçait également son intention de distribuer aux grévistes des montants hebdomadaires anormalement élevés, soit de $\$ 300$ et de $\$ 160$ respectivement pour les employés à plein temps et à temps partiel.

\section{QUESTIONS SOULEVÉES}

La preuve relative aux supposés actes de vandalisme du Syndicat était-elle pertinente? Quels sont les objectifs du mandat conféré au Conseil par le législateur, par l'entremise de la discrétion ministérielle, en vertu de l'article 171.1 du Code, et surtout, sur quelles prémisses le Conseil doit-il se baser pour s'acquitter de son mandat?

La Charte canadienne des droits et libertés, en consacrant la liberté d'association, pourrait-elle rendre inopérant l'article 171.1?

Enfin, un groupe d'employés hostiles au Syndicat avait-il le droit d'être entendu par le Conseil?

\section{PRINCIPAUX MOTIFS DE DÉCISION}

Les objectifs de l'article 171.1 du Code canadien

Le Conseil a d'abord rappelé que le principe fondamental du Code canadien, principe qui prévaut d'ailleurs dans l'ensemble des systèmes de relations industrielles au pays, est la reconnaissance du droit des travailleurs de se grouper pour négocier collectivement leurs conditions de travail et qu'un corollaire de ce principe est que ces travailleurs peuvent légitimement s'attendre à conclure un tel contrat avec leur employeur.

In 1973 [...] Parliament restructured its labour law by introducing a totally revised Part V-Industrial Relations into the Canada Labour Code and by reconstituting the Canada Labour Relations Board. The Preamble of the new Part $V$ of the Code clearly indicates Parliament's desire to encourage collective bargaining as a matter of public policy [...]

Five years later, in 1978, Parliament found it necessary to again introduce major amendments to the Code [...]

'[...] Establishment of first agreements: Very frequently there is a disproportionate power between a newly certified union and the employer. Employers have opposed unionization and have maintained that attitude during negotiation making a first collective agreement impossible. Employers then merely wait in the hope that the union will collapse [...]' 
(The Horourable John Munro, Minister of Labour, Minutes of Proceedings and Evidence of the Standing Committee on Labour, Manpower and Immigration, 3rd Session, 30th Parliament, March 16, 1978, at pages 11:48 to 11:50) [...] 1 .

It was not merely coincidence that Parliament took steps to bring in first contract settlement provisions in 1978 while it was shoring up the certification process. The two are intrinsically linked. It can be seen from the foregoing review that section 171.1 has more to do with the reinforcement of the certification process than it has to do with the settlement of contract provisions in a compulsory arbitration sense. The settlement of first collective agreements by the Board was primarily intended to give support and some meaning to the exercice of the fundamental freedom of association rights of employees $[. . .]^{2}$

Le Conseil, en passant en revue sa jurisprudence relative à l'article 171.1, démontre d'ailleurs qu'il n'a exercé son pouvoir discrétionnaire d'imposer une première convention collective que dans les cas où la preuve avait révélé une attitude fondamentalement anti-syndicale de la part de l'employeur. Il y a une distinction entre cette dernière situation et celle où l'employeur décide d'adopter une ligne dure durant les négociations, ce qui est une stratégie peut-être discutable, mais légale ${ }^{3}$.

De plus, le Conseil a estimé que dans le secteur bancaire, il fallait appliquer l'article $\mathbf{1 7 1 . 1}$ en tenant compte du contexte des relations industrielles dans cette industrie, qui ne sont pas des plus harmonieuses, et il réfère à sa décision commentée plus haut dans cette chronique, soit l'affaire de la Banque Nationale du Canada d̀ Rimouski ${ }^{4}$.

Preuve relative aux actes de vandalisme

Le Conseil a refusé d'entendre cette preuve. La Banque voulait ainsi démontrer que de par son attitude, le Syndicat ne pouvait se voir bénéficier du remède de l'article 171.1. Le Conseil a statué qu'un tel renvoi dépendait uniquement de la discrétion du Ministre et qu'il ne saurait y avoir dans cette procédure une partie requérante qui porterait le fardeau de la preuve. Le Conseil a cependant déclaré qu'un «portrait général» de la conduite du Syndicat tout au long des négociations serait pertinent si il y avait preuve que cette conduite avait influencé l'attitude de la Banque à la table des négociations. Ce n'était pas le cas.

1 Décision originale, pages $5,6,7$.

2 Ibid., page 14.

3 CJMS Radio Montréal Ltee (1978), 27 di 796; [1979] 1 Can LRBR 332; Royal Bank of Canada, (1980), 41 di 199; [1982] 1 Can LRBR 16; MacLean-Hunter Cable TV Limited, (1980), 42 di 274; [1981] 1 Can LRBR 454; General Teamsters Local Union 979 and Gardewine \& Sons Ltd., dossier du Conseil 675-14; Raoul Sénécal Transport Ltée et l'Union des Employés du Transport Local et Industries diverses, Local 931, dossier du Conseil 675-15; Verreault Navigation Inc. and The Seafarers' International Union of Canada, dossier du Conseil 675-16; Fort Alexander Indian Band, décision non rapportée no 462.

4 Syndicat des employés des Banques nationales de Rimouski (CSN) v. Banque nationale du Canada, décision non rapportée no 542. 
Le règlement de la convention collective

Le Conseil a décidé dans ce cas d'imposer une convention aux parties. Il a été convaincu par la preuve que la Banque n'était aucunement préparée à faire quelque concession quant aux questions cruciales, ce qui s'inscrivait dans la tradition des négociations collectives dans ce secteur:

In effect, collective bargaining never really changed the life of bank workers at the CIBC. The bank used its superior bargaining power to restrict even the most rudimentary involvment of the UBE in the affairs of the employees it represents. The bank has retained virtually unrestricted freedom to deal with its employees individually.

In these present negotiations, the bank was not prepared to give one inch in any of those crucial areas [...] [...] We are aware that we have no complaint before us under section 148(a) of the Code, indeed, no such complaint can be brought without Ministerial consent under section 187(5). We therefore make no finding that the bank has contravened section 148(a), but to fulfill our role under section 171.1, we must surely look to the motives behind bargaining postures to determine whether the circumstances call for the remedy Parliament intended. As we said before, after considering all of the evidence and testimony before us we were convinced that the CIBC's short term plan was to keep the union impotent with the long term goal of operating its business without the encumberance of collective bargaining $[. . .]^{5}$

Quant aux termes mêmes de l'accord à être imposé, le Conseil a d'abord précisé que, quant aux questions salariales, il tiendrait compte de la réalité du conflit: le Syndicat avait perdu sa grève:

[...] When we were considering monetary benefits we placed a restriction on ourselves. The reality of the situation was that the union had taken the bank on and had lost. After six months of applying sanctions the union had failed to dent the bank's armour, even with the extra financial support for ihe strikers. In settling economic items we were cautious not to give the union what it had not been able to win through the collective bargaining process. ${ }^{6}$

Mais, relativement aux autres clauses, telles les questions de promotions, mises à pied et droits de gérance, le Conseil a tenu à ce que la convention réflète la légitime place du Syndicat en tant que partie égale.

De plus, le Conseil a incorporé a la convention collective les dispositions du Code canadien du travail concernant les changements technologiques et élargi la juridiction de l'arbitre concernant les griefs.

Enfin, il a également refusé d'endosser un accord des parties visant à exclure certains employés de la portée de la convention: une telle entente restreindrait l'unité de négociation décrite au certificat d'accréditation, par là contraire à l'ordre public ${ }^{7}$.

5 Décision originale, pages $46,47$.

6 Ibid., pages 50, 51 .

7 Voir à ce sujet la décision du Conseil dans British Columbia Telephone Company (1977), 22 di 507; [1977] 2 Can LRBR 404; 77 CLLC 16,108. 
Dans le protocole de retour au travail, partie intégrale de la convention, le Conseil n'a pas eu à débattre de la priorité à donner aux grévistes sur ceux qui les avaient remplacés pendant le conflit, ceci étant accepté par les parties. Cependant, il a réitéré que cette priorité est commandée par le Code canadien du travail.

Mais il a dû statuer sur la question du droit de l'employeur de prendre des sanctions disciplinaires à l'endroit de certains employés relativement aux délits commis sur les lignes de piquetage. Tout d'abord, le Conseil a rappelé sa propre absence de juridiction quant à l'imposition de telles sanctions. Quant à la juridiction de l'employeur, il la met fortement en doute.

[...] One thing that surely cannot be in doubt though is that the construction of section 184(3)(a)(vi) anticipates a restriction on discipline for participation in a lawful strike [...]

In Graham Cable TV/FM (1985), unreported Board decision no. 529, the Board recently said an employer had contravened section 184(3)(a)(vi) by disciplining employees for participating in work slow down tactics which the Board found to be lawful strike activities.

Some sound labour relations reasons why employers should not have authority to discipline employees during a strike, even for unlawful activities, were discussed in another decision which has just been released by the Board (see Pacific Western Airlines Ltd. (1986), unreported Board decision no. 562). ${ }^{8}$

Le Conseil a donc imposé aux deux parties un moratoire sur les représailles, celles-ci étant définies comme comprenant les mesures disciplinaires. En fait, il a soutenu que sans un telle clause d'amnistie, la raison d'être de l'article 171.1, soit la promotion de saines relations de négociation collective, pourrait être bafouée.

La Charte des droits et libertés

La Banque avait soutenu dans sa plaidoirie que l'article 171.1 contrevenait aux articles 2(d), 7 et 15 de la Charte canadienne des droits et libertés.

Libertés fondamentales

2. Chacun a les libertés fondamentales suivantes:

[...]

d) liberté d'expression.

Garanties juridiques

7. Chacun a droit à la vie, à la liberté et à la sécurité de sa personne; il ne peut être porté atteinte à ce droit qu'en conformité avec les principes de justice fondamentale. 
Droits à l'égalité

15. (1) La loi ne fait exception de personne et s'applique également à tous, et tous ont droit à la même protection et au même bénéfice de la loi, indépendamment de toute discrimination, notamment des discriminations fondées sur la race, l'origine nationale ou ethnique, la couleur, la religion, le sexe, l'age ou les deficiences mentales ou physiques.

(2) Le paragraphe (I) n'a pas pour effet d'interdire les lois, programmes ou activités destinés à améliorer la situation d'individus ou de groupes défavorisés, notamment du fait de leur race, de leur origine nationale ou ethnique, de leur couleur, de leur religion, de leur sexe, de leur âge ou de leurs déficiences mentales ou physiques.

En regard de l'article $2 \mathrm{~d}$ ), la Banque alléguait que les termes «liberté d'association» englobaient le pouvoir d'un employeur de diriger à sa façon son entreprise, ceci étant le «sine qua non» de l'existence de l'entreprise.

Tout d'abord le Conseil a désapprouvé cet argument en statuant que l'essence de l'article $\mathbf{1 7 1 . 1}$ était plus de renforcer le processus d'accréditation que de déterminer les termes d'une convention collective. Cependant, puisque la Cour suprême dans l'affaire $R$. v. Big $M$. Drug $\operatorname{Mart}^{9}$, a énoncé que non seulement le but mais aussi l'effet d'une loi doivent être considérés pour déterminer leur validité en regard de la Charte, le Conseil s'est demandé si l'article 171.1 avait pour effet d'affecter la liberté d'association.

Aux Etats-Unis, lors de la période qu'on a par la suite surnommée «l'ère Lochner $",{ }^{10}$ les cours avaient statué que le règlement statutaire des conditions de travail était inconstitutionnel. Cette tendance fut cependant renversée en 1937 par la Cour suprême ${ }^{11}$.

Constatant l'abandon de ce courant jurisprudentiel, le Conseil a conclu au surplus qu'il se fondait sur la liberté contractuelle plutôt que sur la liberté d'association.

The bank cannot give greater force to its constitutional argument by trying to dress up what is really an argument about freedom of contract into an argument about freedom of association... ${ }^{12}$

Se penchant sur la liberté d'association, le Conseil a passé en revue les décisions canadiennes rendues à ce sujet en vertu de la Charte. Il en a conclu que si les juges ne s'entendent pas sur la définition de l'action collective, ils s'accordent cependant sur celle de l'association, qui se lit ainsi:

An association is the coming together of individuals to promote their mutual interests. In that light it is quite clear that freedom of contract involves something which is fundamentally different. Classically, when two parties enter into a contract they are not acting in concert to promote their mutual interests, Rather, they are two parties on opposite sides of a bargain each trying independently to promote their own separate interests...

$9 \quad$ R. v. Big M Drug Mart (1985), 18 D.L.R. (4th) 321 (S.C.C.).

10 Lochner v. New York, 198 U.S. 45 (1905).

11 West Coast Hotel Co. v. Parrish, 300 U.S. 379 (1937).

12 Décision originale, page 67. 
[...] The fact that some associations may come together by means of a contract cannot turn contractual arrangements generally into circumstances in which freedom of association applies. ${ }^{13}$

Le Conseil a donc conclu que la liberté revendiquée par la Banque était celle de contracter et non pas celle de libre association. La première n'étant pas protégée par la Charte, il a rejeté l'argument de la Banque. Mais celle-ci revenait à l'assault en brandissant l'article 7 de la Charte.

Le Conseil a rejeté la thèse de la Banque voulant que le mot «liberté» inclue la liberté économique en général ou la liberté de contracter en particulier, l'ensemble des autorités n'appuyant pas cette proposition ${ }^{14}$.

Enfin, le Conseil a également refusé d'accepter l'argument de la Banque à l'effet que l'article $171.1 \mathrm{du}$ Code canadien du travail enfreignait l'article 15 de la Charte.

La Banque soutenait qu'en lui imposant les termes d'une convention collective, le Conseil lui assénait un traitement particulier, ne respectant pas ainsi le principe d'égalité devant la loi édictée par l'article 15 de la Charte. Le Conseil a constaté que l'article 171.1 était une disposition d'application générale, qui ne visait aucunement la Banque de façon particulière, et conséquemment, ne pouvait être rendu inopérant par le biais de l'article 15 de la Charte.

Le droit d'un groupe d'employés d'être entendus

Dans un excès de prudence, dû à la récente décision de la Cour fédérale dans Okanagan Helicopters ${ }^{15}$, le Conseil avait avisé de l'audition tous les employés des unités concernées. Il a cependant refusé de leur reconnaître l'intérêt nécessaire pour faire des représentations et a également, après avoir constaté cette absence d'intérêt, refusé d'exercer son pouvoir discrétionnaire d'entendre leurs représentations.

Le Conseil explique l'absence d'intérêt des employés en l'instance de la façon suivante: tout d'abord, il était clair de par leurs représentations que le groupe d'employés voulant être entendu s'opposait non pas au règlement de la convention collective par le Conseil, mais au Syndicat. Il désirait que le certificat d'accréditation soit amendé de façon à exclure de l'unité les employés mécontents. Le Conseil a écarté cette proposition:

[...] Mr. Manning was attacking head on the notion of the exclusive bargaining agent system based on majority rule. He was inviting us to undo more than 40 years of history of labour statutes in this country...

13 Ibid., pages $70,71$.

14 Public Service Alliance of Canada v. The Queen in Right of Canada (1984), 11 D.L.R. (4th) 337 (F.C., T.D.); Manion et al. v. County of Oxford et al. (1985), 52 O.R. (2d) 137 (Ont. Div. Ct.).

is Okanagan Helicopters Ltd. v. Canadian Helicopter Pilot's Association and CLRB (1986) 86 CLLC 14,007. 
[...] Mr. Manning was seeking to transform the section 171.1 proceeding into something quite different, i.e., into a section 119 review proceeding... ${ }^{16}$

Mais le fondement principal du Conseil pour dénier aux employés un intérêt aux procédures fut plutôt les articles 107(1) et 171.1 du Code.

Après avoir statué que dans le cadre des procédures sous l'article 171.1, le paragraphe (a) de 107(1) s'appliquait, le Conseil a constaté que les employés brillaient par leur absence dans la définition de «parties».

Par ailleurs, le paragraphe (2) de 171.1 indique clairement que les employés de l'unité et les «parties» sont deux choses distinctes.

Au surplus, le paragraphe (3) de l'article 171.1 stipule clairement que le Conseil n'a l'obligation d'entendre que les «parties». Ceci découle du principe bien établi qu'un syndicat accrédité détient le pouvoir exclusif de négocier collectivement au nom des employés de l'unité (voir 136(1)e) C.c.t.). Dans l'affaire Bibeault ${ }^{17}$, la Cour suprême a reconnu que les employés n'avaient pas l'intérêt requis pour être entendus lors de la détermination du caractère approprié d'une unité de négociation.

Cependant, le Conseil a reconnu que dans certaines circonstances, des employés pourraient accéder au statut de partie. Ces circonstances surviendraient lorsqu'un syndicat prendrait la cause d'un groupe d'employés au détriment d'un autre, ceci conférant alors à ce dernier groupe l'intérêt et le droit d'être entendu ${ }^{18}$.

Cependant, le Conseil a estimé que le simple fait qu'un groupe d'employés n'appuie pas le syndicat n'est pas suffisant pour conférer à ce groupe un intérêt contraire à et distinct de celui du syndicat qui en ferait une «partie».

Non-support of the union or its position is not sufficient to make an employee's interest legally adverse so as to entitle the employee to be a separate party. The common interest shared by the employees who sought to make representations before the Board in the present case was that they did not support the UBE. But that was not the foundation on which the union sought to, or could have sought to, base any differential treatment in any term of the collective agreements settled by the Board. There is no term in the collective agreement which singles out union supporters for different treatment compared to non-supporters. ${ }^{19}$

\section{DECISION}

Le Conseil a réglé les modalités de la première convention collective entre la Banque et le Syndicat.

16 Décision originale, pages $78,79$.

17 Bibeault et al. v. McCaffrey (1984) 52 N.R. 241 (S.C.C.).

18 Voir Appleton et al. v. Eastern Provincial Airways Ltd. et al., [1984] 1 F.C. 367 (C.A.); Okanagan Helicopters, supra, note 15; Hoogendoorn v. Greening Metal Products and Screening Equipment Company, [1968] S.C.R. 30.

19 Décision originale, page 88. 